\section{A Note on the Stratified Turbulent Flow with no Shear}

\author{
A. K. Chakraborty \\ Nimta High School, Calcutta-700049, India
}

H. P. Mazumdar

Indian Statistical Institute, Calcutta-700035, India

Z. Naturforsch. 40a, 649-650 (1985);

received December 16, 1984

The spectral characteristics of turbulent kinetic energy of a stratified turbulent flow with no shear are described within the framework of similarity theory.

For the equilibrium region $k \gg L_{0}^{-1}$, where $L_{0}$ is a length comparable with the scale of flow as a whole, the balance of kinetic energy in a thermally stratified turbulent flow with no shear yields for the rate of total dissipation $[1,2]$ :

$\varepsilon=G(k)+\frac{g}{\bar{T}} \int_{k}^{\infty} \varphi_{w T}\left(k^{\prime}\right) \mathrm{d} k^{\prime}+2 v \int_{0}^{k} k^{\prime 2} \varphi\left(k^{\prime}\right) \mathrm{d} k^{\prime}$.

In deducing (1), the motion is assumed to be horizontally homogeneous [2].

In this note we treat the effects of buoyancy on the spectral characteristics of turbulent kinetic energy in isolation, and for that we impose the following restrictions:

(a) the interaction between the mean and turbulent fields is weak,

(b) $k \ll k_{\mathrm{d}}\left[=\left(\varepsilon / v^{3}\right)^{1 / 4}\right]$ so that the viscous dissipation term in (1) may be neglected.

(c) $k$ is sufficiently large so that the turbulence is not too remote from isotropy [3].

(d) The production of thermal energy does not affect the mechanical turbulence.

Under the above assumptions, (1) takes the form

$$
\varepsilon=G(k)+\frac{g}{\bar{T}} \int_{k}^{\infty} \varphi_{w T}\left(k^{\prime}\right) \mathrm{d} k^{\prime},
$$

where $\int_{0}^{\infty} \varphi_{w T}(k) \mathrm{d} k=\frac{k}{w T^{\prime}}, T^{\prime}$ being the fluctuation of temperature. For the term $G(k)$, which represents the transfer of energy through the hierarchy of eddies, we use the forms due to Kovasznay [4] and Pao [5] separately. The buoyancy term, i.e. the second term on the r.h.s. of (2) is modelled appropriately in each case.

Case i): According to Kovasznay [4]

$$
G(k)=\alpha^{-3 / 2} k^{5 / 2}[\varphi(k)]^{3 / 2},
$$

where $\alpha$ is the Kolmogroff constant. Relation (3) may be interpreted as

$$
G(k)=v_{T}^{K}(\text { vorticity })^{2},
$$

Reprint requests to Herrn A. K. Chakraborty, 61, Anjangarh, Birati, Calcutta-700051, Indien. where $v_{T}^{K}$ is the transport coefficient given by

$$
v_{T}^{K}=\alpha^{-3 / 2} k^{-1 / 2}[\varphi(k)]^{1 / 2} .
$$

The vorticity, as determined from dimensional considerations is given by $k^{3 / 2}[\varphi(k)]^{1 / 2}$. Under the assumption (a), the buoyancy term can be written [1] as

$$
\frac{g}{\bar{T}} \int_{k}^{\infty} \varphi_{w}\left(k^{\prime}\right) \mathrm{d} k^{\prime}=-v_{T}^{*}\left(\frac{g}{\bar{T}} \frac{\mathrm{d} \bar{T}}{\mathrm{~d} z}\right),
$$

where $v_{T}^{*}=f v_{T}^{K}, f$ being a constant. It is to be noticed that $\frac{g}{\bar{T}} \frac{\mathrm{d} \bar{T}}{\mathrm{~d} z}$ has the same dimension as the square of the vorticity. We assume $\frac{\mathrm{d} T}{\mathrm{~d} z}>0$, which indicates that the region of the free atmosphere concerned is stably stratified.

In view of the relations (3), (5) and (6), the solution of (2) is obtainable in the non-dimensional form

$$
\begin{aligned}
\hat{\varphi}=2^{-2 / 3} \alpha \hat{k}^{-5 / 3} & {\left[\left\{\left(1-\frac{4}{27 \alpha^{3} \hat{k}^{4}}\right)^{1 / 2}+1\right\}^{1 / 3}\right.} \\
& \left.+\left\{1-\left(1-\frac{4}{27 \alpha^{3} \hat{k}^{4}}\right)^{1 / 2}\right\}^{1 / 3}\right]^{2}, \quad \hat{k}>\left(\frac{4}{27 \alpha^{3}}\right)^{1 / 4},
\end{aligned}
$$

where $\hat{k}=k /\left(b^{3 / 4} \varepsilon^{-1 / 2}\right), \quad \hat{\varphi}=\varphi /\left(b^{-5 / 4} \varepsilon^{3 / 2}\right), \quad$ and $\quad b$ $\left(=f \frac{g}{\bar{T}} \frac{\mathrm{d} \bar{T}}{\mathrm{~d} z}\right)$ is a dimensional parameter.

Case ii): According Pao [5]

$$
G(k)=\alpha^{-1} \varepsilon^{1 / 3} k^{5 / 3} \varphi(k) .
$$

As before, $G(k)$ can be expressed in the form (4), where the transport coefficient $v_{T}^{P}$ (instead of $v_{T}^{K}$ ) is given by

$$
v_{T}^{P}=\alpha^{-1} \varepsilon^{-1 / 3} k^{1 / 3} \varphi(k)
$$

and the vorticity is given [6] by $\varepsilon^{1 / 3} k^{2 / 3}$. The buoyancy term in this case is modelled as

$$
\frac{g}{\bar{T}} \int_{k}^{\infty} \varphi_{w T}\left(k^{\prime}\right) \mathrm{d} k^{\prime}=-f^{*} \cdot v_{T}^{P}\left(\frac{g}{\bar{T}} \frac{\mathrm{d} \bar{T}}{\mathrm{~d} z}\right),
$$

where $f^{*}$ is another constant. In view of the relations (8), (9) and (10) the solution of (2) is obtainable in non-dimensional form

$$
\tilde{\varphi}=\alpha /\left(\tilde{k}^{5 / 3}-\tilde{k}^{1 / 3}\right), \quad \tilde{k}>1,
$$

where $\tilde{k}=k /\left(b^{* 3 / 4} \varepsilon^{-1 / 2}\right), \quad \tilde{\varphi}=\varphi /\left(b^{*-5 / 4} \varepsilon^{3 / 2}\right), \quad$ and $b^{*}$ $\left(=f^{*} \frac{g}{\bar{T}} \frac{\mathrm{d} \bar{T}}{\mathrm{~d} z}\right)$ is a dimensional parameter.

Choosing the Kolmogoroff constant $\alpha=1.70$, we plot $\log \hat{k}$ vs. $\log \hat{\varphi}$ (Fig. 1) and $\log \tilde{k}$ vs. $\log \tilde{\varphi}$ (Figure 2 ).

Essentially, the turbulence considered is decaying, it has been formed at some time in the past by wind shear or flux divergence, but is now being damped by working against the buoyancy force of stable stratification.

In case i), for a small portion of the equilibrium range, the energy spectrum follows a $-11 / 5$ power dependency on the wave number (Figure 1). Bolgiano [7] termed this wave number range as buoyancy subrange. In case ii), the gradient of the spectrum in such a buoyancy subrange is approximated in different portions by exponential relations with exponents $-11 / 5$ and -3 (cf. Lumley [8] and

0340-4811/ 85 / 0600-0649 \$ 01.30/0. - Please order a reprint rather than making your own copy. 


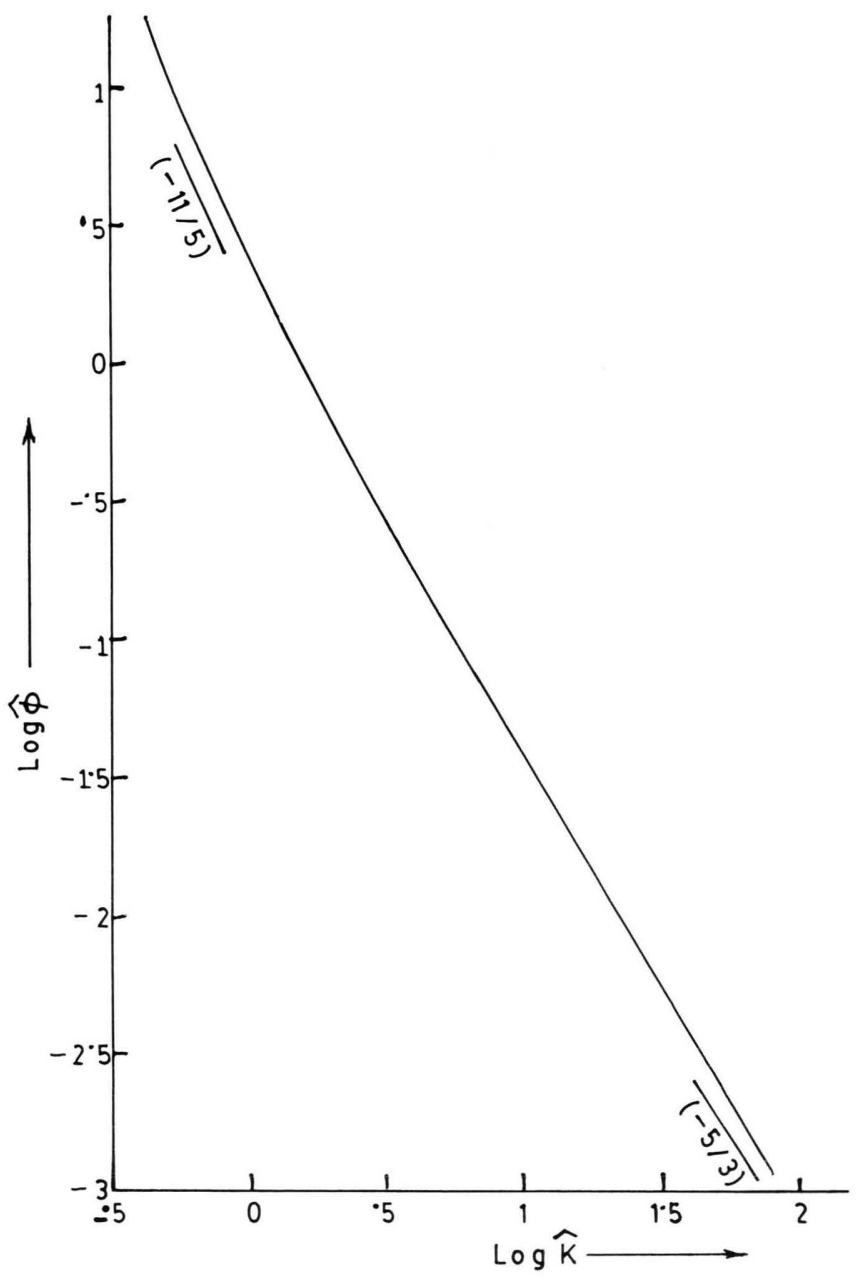

Fig. 1.

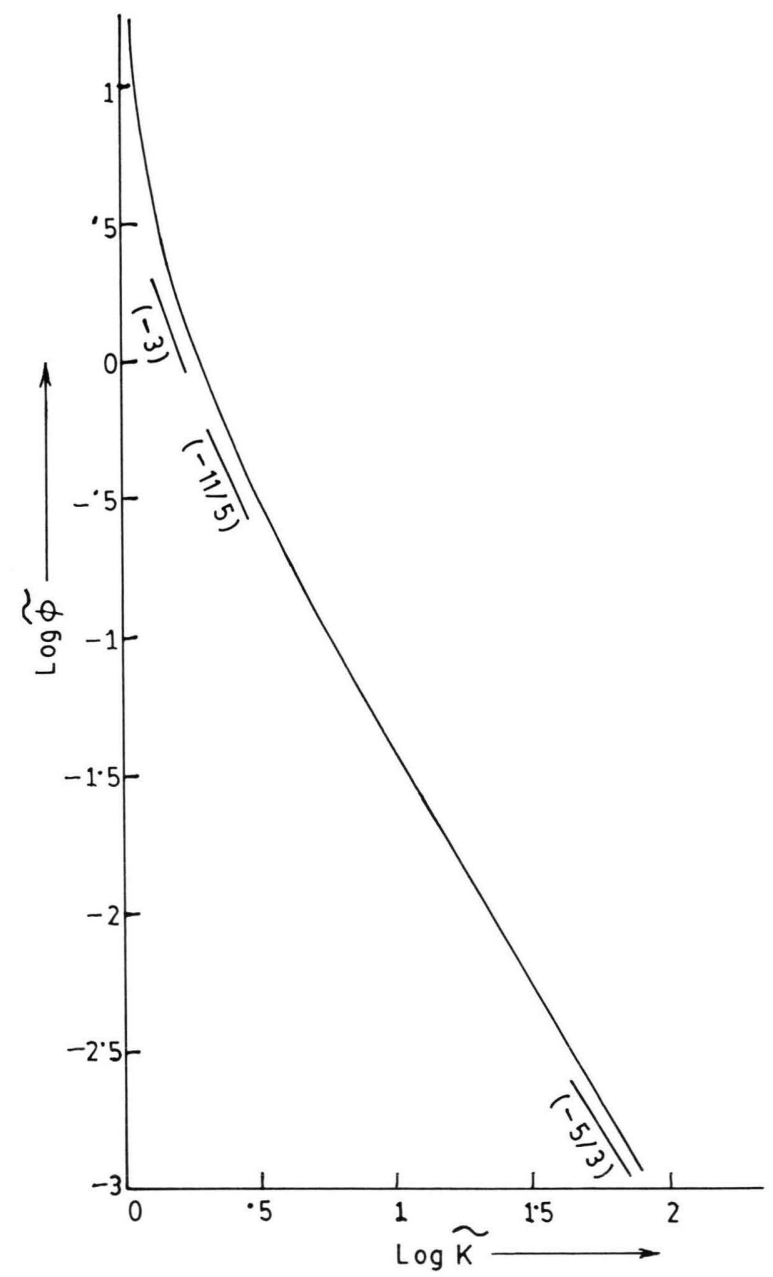

Fig. 2.
[9]). In the microscale turbulence region of the upper troposphere and lower stratosphere, the aircraft measurements of Pinus [10] showed that the modulus of the slope of the spectral density curve exceeds $5 / 3$. Also Vinnichenko et al. [11] observed at altitudes of $6 \mathrm{~km}$ and $9 \mathrm{~km}$ stable thermal stratifications with small vertical gradients of mean wind velocity.

The treated energy spectum exhibits at higher wave numbers the classical $-5 / 3$ law (Fig. 1 and 2). Such "minus five thirds" behaviour exists in the atmosphere over a range of eddy size below $200 \mathrm{~m}$ [12].

\section{Acknowledgements}

The authors are thankful to Professor L. N. Persen, Norway, Professor A. Ghosh, Calcutta, Professor K. Bagchi, Jadavpur, and Professor K. M. Ghosh, Calcutta for stimulating discussions on this problem.

[7] R. Bolgiano, J. Geophys. Res. 64, 2226 (1959).

[8] J. L. Lumley, J. Atmos. Sci. 21, 99 (1964).

[9] G. N. Shur, Trudy TsAO 43, 79 (1962).

[10] N. Z. Pinus, Meteorol. Gidrol. No. 4, 3 (1966).

[11] N. K. Vinnichenko et al., Atmospheric Turbulence and Radio Wave Propagation (Coll. in Moscow), Nauka, Moscow 1965, p. 65.

[12] E. R. Reiter and A. Burns, J. Atmos. Sci. 23, 206 (1966).

4] L. S. G. Kovasznay, J. Aeronaut. Sci. 15, 745 (1948).

[5] Y. H. Pao, Phys. Fluids 8, 1063 (1965).

[6] S. Panchev, Random Functions and Turbulence, Pergamon Press, Oxford 1971, p. 218. 